\title{
Characterization and promoter activity of chromoplast specific carotenoid associated gene $(C H R C)$ from Oncidium Gower Ramsey
}

\author{
Chung-Yi Chiou $\cdot$ Keqiang Wu $\cdot$ Kai-Wun Yeh
}

Received: 9 May 2008/Revised: 16 May 2008/Accepted: 29 May 2008/Published online: 25 June 2008

(C) Springer Science+Business Media B.V. 2008

\begin{abstract}
Tissue-specific promoters are required for plant molecular breeding to drive a target gene in the appropriate location in plants. A chromoplast-specific, carotenoid-associated gene $(\mathrm{OgCHRC})$ and its promoter (Pchrc) were isolated from Oncidium orchid and characterized. Northern blot analysis revealed that $O g C H R C$ is specifically expressed in flowers, not in roots and leaves. Transient expression assay of $P$ chrc by bombardment transformation confirmed its differential expression pattern in floral tissues of different horticulture plants and cell-type location in conical papillate cells of adaxial epidermis of flower. These results suggest that Pchrc could serve as a useful tool in ornamental plant biotechnology to modify flower color.
\end{abstract}

Keywords Carotenoids - Flower colour . Orchid (Oncidium) · Plant pigments .

Tissue-specific promoter

\section{Introduction}

In plants, carotenoid pigments are essential in pollinators and other organisms, photoprotection

C.-Y. Chiou · K. Wu · K.-W. Yeh $(\bowtie)$

Institute of Plant Biology, College of Life Science,

National Taiwan University, Taipei 106, Taiwan

e-mail: ykwbppp@ntu.edu.tw and UV-B radiation protection (Stracke et al. 2007; Naik et al. 2003). CHRC is a chromoplast-specific, carotenoid-associated protein that functions in sequestration and accumulation of carotenoid in plastid membranes during flower and fruit development (Vishnevetsky et al. 1999b). Due to the association with CHRC protein, carotenoids are stabilized and accumulated in membranes to a considerable level. A cucumber $C H R C$ gene and its promoter activity have been intensively studied. Cucumber CHRC gene displays spatial expression in floral tissues and temporal activation in parallel to floral development (Vishnevetsky et al. 1996, 1999a).

Oncidium Gower Ramsey hybrid has become a popular cut flower in Asian markets. The yellow pigment in floral tissues is comprised of carotenoid compounds (Hieber et al. 2006). However, the simple yellow floral color seems not to meet customers' demands. Modification of floral pigments by engineering pigment-biosynthesizing genes could be a useful strategy to change flower color (Zuker et al. 2002). An eligible promoter that can drive gene expression specifically in parallel to floral development, rather than a constitutive one such as CaMV $35 S$ promoter, is a crucial component to successfully drive pigment-biosynthesizing genes in floral tissue cells. Based on this motivation, $\mathrm{CHRC}$ gene $(\mathrm{OgCHRC})$ and promoter sequence (Pchrc) were isolated from floral lip of Oncidium orchid and characterized. To delineate the differential function of $C H R C$ promoter region and the expression pattern, bombardment 
transformation assay was carried out by using GUSfusion system in various plant species. The results clearly showed that Pchrc was specifically expressed in the conical papillate cells of adaxial epidermis of floral tissues. Our data strongly supports that Pchrc of Oncidium could serve as an effective promoter to drive pigment-biosynthesizing genes and to modify floral pigments in plant biotechnology.

\section{Materials and methods}

Plant materials and RNA isolation

Oncidium Gower Ramsey were obtained from HsinTung Orchid Nursery. Taoyuan, Taiwan. The floral buds of different developmental stages and floral lips were harvested for RNA isolation and for biolistic bombardment.

Cloning of Oncidium CHRC and its promoter region

Poly(A) ${ }^{+}$RNA was purified from total RNA using Oligotex mRNA Kit (Qiagen).

The 5'- and 3'-Rapid Amplification of cDNA Ends ( $5^{\prime}$ - and $3^{\prime}$-RACE) on CHRC genes was performed (Clontech) according to the manufacturer's instruction. The primer sequences for the amplification of full length cDNA were as the followings:

Forward: $5^{\prime}$-ACCGGGAGTTTTCAGAAAAA-3'; Reverse: 5'-TTTGATTCGGACTGTAATATTT-3'.

The promoter region of $O g C H R C$ was amplified from genomic DNA template by genome-walking method (GenomeWalker Universal Kit, Clontech). The primer sequences for the amplification of $1.7 \mathrm{~Kb}$ fragment Pchrc were as the followings: Forward: $5^{\prime}$ GTCGTGAGATATTCTCATTTGCTG-3'; Reverse: 5'-GTTTGAACTTGGGAGTTGGGG-3' .

Northern blot analysis

Total RNA was isolated from flower bud by phenol/ chloroform extraction and $\mathrm{LiCl}$ precipitation (Chang et al. 1993). Ten $\mu \mathrm{g}$ RNA of each sample was resolved on $1 \%$ denatured/formaldehyde agarose gel, transferred onto a Immobilon- $\mathrm{N}^{+}$membrane (Millipore), and UV cross-linked. The membranes were hybridized at $65^{\circ} \mathrm{C}$ with $\alpha-{ }^{32} \mathrm{P}-\mathrm{dCTP}$-labeling probe.
Following hybridization, membranes were washed twice at room temperature in $2 \times$ SSC containing $0.1 \%$ SDS for $15 \mathrm{~min}$ and once in $0.1 \times \mathrm{SSC}$ at $60^{\circ} \mathrm{C}$ for $10 \mathrm{~min}$.

Constructs for transient expression and particle bombardment

DNA cassettes of GUS gene fused with Pchrc or CaMV 35S promoter in pCAMIA1390 vector were amplified by PCR and subcloned into a pGEM-T easy vector. The purified recombinant plasmid DNA was prepared for bombardment transformation to floral tissues. The petal tissues of Oncidium, Rose, Lily, Carnation and Chrysanthemum were freshly detached. Bombardment assay was conducted following the method described by Vishnevetsky et al. (1999), using the instrument of Helium Biolistic particle Delivery System (Model PDS-100, BioRAD). In brief, plasmid DNA ( $1 \mu \mathrm{g})$ was precipitated with $0.6 \mathrm{mg}$ gold particles $(1.0 \mu \mathrm{m}$ diameter $)$ through the addition of $10 \mu \mathrm{l} 2.5 \mathrm{M} \mathrm{CaCl}_{2}$ and $4 \mu \mathrm{l} 100 \mathrm{mM}$ spermidine. After precipitation, the particles were washed twice with absolute ethanol, and resuspended in $20 \mu \mathrm{l}$ absolute ethanol. Then, the particles were pipetted onto a microcarries of the Biolistics Device. For bombardment, lip tissue was placed on $0.5 \times \mathrm{MS}$ medium with $7.5 \%$ agar in a Petri dish, and was bombarded at a distance of $9 \mathrm{~cm}$ from the stopping plate using $900 \mathrm{psi}(1 \mathrm{psi}=6.89 \mathrm{kPa})$ rupture disks. Bombarded tissues were subsequently incubated on MS medium at $22^{\circ} \mathrm{C}$, under a $16 \mathrm{~h}$-light $/ 8 \mathrm{~h}$-dark photoperiod condition for 2 days.

\section{Histochemical analysis of GUS expression}

The bombarded lip were transferred into histochemical reagent containing $0.1 \mathrm{M}$ phosphate buffer, $0.5 \mathrm{mM}$ ferricyanide, $0.5 \mathrm{mM}$ ferrocyanide, $0.1 \%$ Triton X-100, $10.0 \mathrm{mM}$ EDTA, 20\% (v/v) methanol and $1 \mathrm{mM}$ 5-bromo-3-indolyl-glucuronide (Sigma). The samples were incubated for 2 days at $37^{\circ} \mathrm{C}$. After staining, the samples were cleared with $70 \%(\mathrm{v} / \mathrm{v})$ ethanol and fixed in FAA (45\% absolute ethanol, 5\% glacial acetic acid, 5\% formaldehyde). The microscopic observation was carried out under the contrast phase light microscope. 
GUS fluorometric assay

The bombarded tissues were extracted in $50 \mathrm{mM}$ sodium phosphate buffer $\mathrm{pH} 7.0$, containing $0.1 \%$ Triton X-100, $10 \mathrm{mM}$ EDTA, and $10 \mathrm{mM} \beta$-mercaptoethanol. Following centrifugation, extracts was incubated with the same buffer containing $1 \mathrm{mM} 4$ methylumbelliferyl $\beta$-D-glucuronide (MUG). Aliquots were withdrawn after $0,15,30$ and $45 \mathrm{~min}$, the reaction was stopped with $0.2 \mathrm{M} \mathrm{Na}_{2} \mathrm{CO}_{3}$. Six replicates for each sample were measured with a Fluoroskan Ascent FL fluorometer (Labsystems). The protein concentration of each sample was determined using Quick Start Bradford Protein Assay kit (Bio-Rad).

\section{Results and discussion}

Molecular characterization of a chromoplast specific carotenoid-associated protein gene, OgCHRC

By using of RT-PCR and $5^{\prime} / 3^{\prime}$ RACE, an $O g C H R C$ cDNA (GeneBank access NO. EU583501) of 1,069 bp encoding 319 deduced amino acid residues was successfully isolated. The protein sequence analysis revealed that OgCHRC shares 68\%, 67\% and $66 \%$ identity with CHRC of Arabidopsis thaliana, Oryza sativa and Cucumis sativus $L$. respectively (Wijk et al. 2006; Cooper et al. 2003; Vishnevetsky et al. 1996).

Northern blot analysis was carried out to examine the pattern of $\mathrm{OgCHRC}$ expression pattern in roots, leaves, flower buds of five developmental stages (S1-S5) and

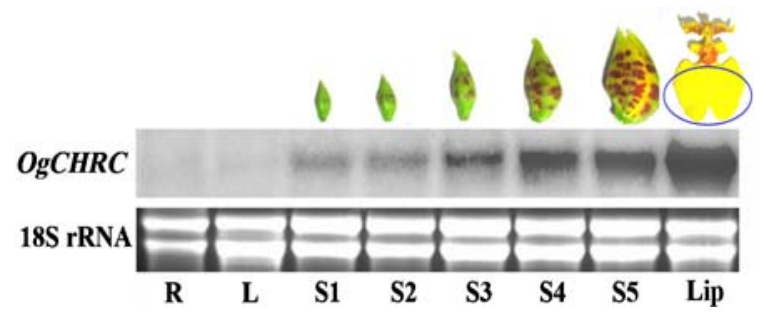

Fig. 1 Northern blot analysis of the $O g C H R C$ gene expression during flower development. Ten $\mu \mathrm{g}$ total RNA from roots, leaves, S1-S5 (five different floral developmental stage) flowers and lips were loaded, respectively. In the lower panel, ethidium bromide-stained ribosomal RNA is shown as an internal control floral lip tissues. The result revealed that the expression level of $\mathrm{OgCHRC}$ increased gradually from S1 to S5 and exhibited a maximal expression level in the lip tissue (Fig. 1). In contrast, the expression level of $O g C H R C$ was not detected in root and leaf tissues, suggesting that $O g C H R C$ is a floral-specific expressed gene.

Isolation and sequence analysis of $\mathrm{OgCHRC}$ promoter (Pchrc) in silico

A 1,703 bp genomic DNA fragment located upstream of the $\mathrm{OgCHRC}$ coding sequence was isolated by using genome-walking protocol. Nucleotide sequence analysis in silico was performed by using various database search programs including PLACE (http://www.dna.affrc.go.jp, Higo et al. 1999), CISTER (http://www.zlab.bu.edu; Frith et al. 2001) and PlantCARE (http://bioinformatics.psb.ugent.be/ webtools/plantCARE; Higo et al. 1999; Prestridge 1991). Sequence analysis indicated that this fragment contained the basal regulatory elements including TATA box for RNA polymerase binding and CAAT box for transcription frequency regulation (Fig. 2). Two 6-bp elements, ATAGAA, that is essential for regulation of plastid genes (Kapoor and Sugiura 1999) were found between nucleotides $-1200 \sim-$ 1194 and $-1002 \sim-998$ bp (Fig. 2). Thus, this DNA fragment could be a functional promoter region of OgCHRC and was designated as Pchrc.

Transient expression analysis of the OgCHRC promoter using particle bombardment

In situ transient expression assay depending on a particle bombardment delivery system has been used to detect promoter efficiency (Vishnevetsky et al. 1999). Two constructs were employed in the transient expression assay. The first one is the $1,703 \mathrm{bp}$ $O g C H R C$ promoter region fused to the $\beta$-glucuronidase gene (Pchrc::GUS), and the other is the CaMV35S promoter driving GUS gene (35S::GUS) as a control. Transient $G U S$ expression driven by the $O g C H R C$ promoter, mimicked the pattern of $O g$ CHRC transcript levels expressed in root, leaf and flower tissues, as well as during different flower development stages (Fig. 3a, b). Pchrc::GUS was preferentially expressed in flower. Histochemical analysis of Pchrc::GUS activity in lip tissue, using 
Fig. 2 Nucleotide sequence of $\mathrm{OgCHRC}$ promoter region. The transcription initiation site is marked as +1 . TATA box and CAAT box are highlighted in bold with green and pink color respectively. Two putative repeated elements (ATAGGA) for regulatory function of plastid genes are highlighted in bold and underlined in red

\begin{abstract}
-1703 GTCGTGAGATATTCTCAT TTGCTGCTCACATACATTGCTTATGACATCTACGT GAAAATTTCA

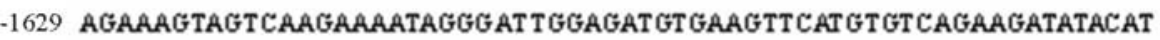

-1567 GGCGGGGAACA GTGATGATATGATCACCTTCAATCGGTTGATGACTGATATAAT TAACATAA

-1505 AGGTAGACGCT TATAAT TGGCTGATTGAATGAGATGTGACAAAGTGGAGCCTACTGAATGA

-1443 CGGTGGAATGCATAGTGGAGCAATGACAACCAATGCTTCAGAATGCTTCAATTCAGTCTTG

-1381 AAGCGAGCTAGATGTTTGCCCATTCAGGCATTAATAATAACAGTT TATTACAATATTATCACA

-1319 ATTTTTTTGAAGCGTTATAAGAGTGCTTCAGAACAGCTTGAGGCCAACGAATCGACATTCGC

-1257 ACAACGCACATTGCGTCAACTCCAACAGTATGGACGAGAAGTTAGGAATTTTCCTTCGCCA

-1195 AT TAACATCAATAGAAGCGAGTTACAAGTGTTGACAAAGACTCAAGATCT TTCCAAGTT

-1135 CaGaATATTCTCCTGCTGARATATGTAGTTGCACTTGTGATAGATGTGAGaTGTATCACATGC

-1073 ctTGTGCTCATGCCATCARAGCTCTTGCAagtagGCGTaTGGTTTATACCACATATGTCTCCA

-1011 GaTaTTAT TCAGTGGACTTCTATAGAAATACCTACACAGAT GaATTCCACACTCTACCTGAT

-949 CGCTCATACTGGCCAGATTGCAACGAAGAAATGAGAT TGATTCAGCTACTACCACCACCAA

-887 ACAGGAAGCGT TCAGGCCGACCACGCTCARATCGATTCGTAACACGATGGACGAGGGTC

-825 CAAGCAAATGTAGATGAGGGATGTCCATTTCAATACCTGCTT TGTAATATTAAACATC TATTT

-763 GGT TAATAAATTGTTCTAGTGTGATGTT TTAATATTTTTGTAATATTGAACATGATGAATT TTC

-701 ATTTCAATTTACTTCATCTTCGTTTCTCTATTTTCATATATTTCTGTCATT TTACATGTTCCAAA

-639 TGAATTGCTACTTACACAT TAAACTACAT TTAT GTATATTT TTCAATATTT TTAATT GATAAAAG

-577 ACATTTT TATCTTCAATT CCATATAARATTTTCTCATTTCAATGCARAAC TTARAATATTATGG

-515 ATTT TCTAACCTARAACCCCGAGAGCARAATGGAAGAGACTAARAARAAARAATAGGAAG

-453 TTAGAAATCGACGACCGTGTCATCGATTTCACGAGAAATCGATTATACGATCGTCGATTCTT

-391 ATARATARGTARAATCGTTT TACCTGTCATCGATTTCTATARAACACTTTTCTAT TACTTTCCT

-329 GCCAACGTGGCATCTGAGCTAGaATGAAATCGGaCTATTAGAGTCCGATTATATCTATTTTTA

-267 AAT TTTTTTTAAT GATACT TATTTTGC TAATTAAATTAT TAATCATACT TATTTTAAAAAT TTGA

-205 CCCARAARACTGCAATCCARACATCCARAATTTTTAGTCAATTCGTTACCTAACACCCCTAA

-143 ATATACARARATTARAACARTGCCCATCCACACARACAATARARARATCTCGGCCCTCGC

-83 ACTCGGTAACTCGGTATTCTTT TAAGCACGTAAAAAT TCCTCATAACATCTATA AAA TAGC -22 TCCCCAACTCCCAAGTTCARAC ${ }^{+1}$
\end{abstract}

the chromogenic substrate X-Gluc, is illustrated in Fig. 4a and b. Pchrc::GUS was specifically expressed in conical papillate cells of adaxial epidermis of lip tissues where carotenoid and anthocyanidin are accumulated (Hieber et al. 2006), suggesting a celltype-specific function. However, the $35 S:: G U S$ did not exhibit such organ-specificity in different tissues of Oncidium as Pchrc (Fig. 3a).

Analysis of OgCHRC promoter activity in floral tissues of various plants

$O g C H R C$ promoter activity was further analyzed in rose, chrysanthemum, carnation and lily. Low activities of $\mathrm{OgCHRC}$ promoter were detected in rose, chrysanthemum and carnation. On the contrary, the activity of $\mathrm{OgCHRC}$ promoter was relative high in lily and Oncidium compared with the other three plant species (Fig. 4). This result indicates that $\mathrm{OgCHRC}$ promoter is more efficient in monocot plants than in dicots. In comparison, the CaMV35S promoter had higher activities in these five plants (Fig. 4).

Oncidium Gower Ramsey is an important and popular cut flower for commercial orchid market. Recently, the flower color genes involving in carotenoid and anthocyanidin biosynthetic pathways have been identified in Oncidium Gower Ramsey (Chiou and Yeh 2008; Hieber et al. 2005). Furthermore, genetic transformation mediated by Agrobacterium tumefaciens and by particle bombardment had been established in Oncidium (Liau et al. 2003; Li et al. 2005). More recently, Chiou and Yeh (2008) successfully induced anthocyanin biosynthesis in yellow lip tissues by 

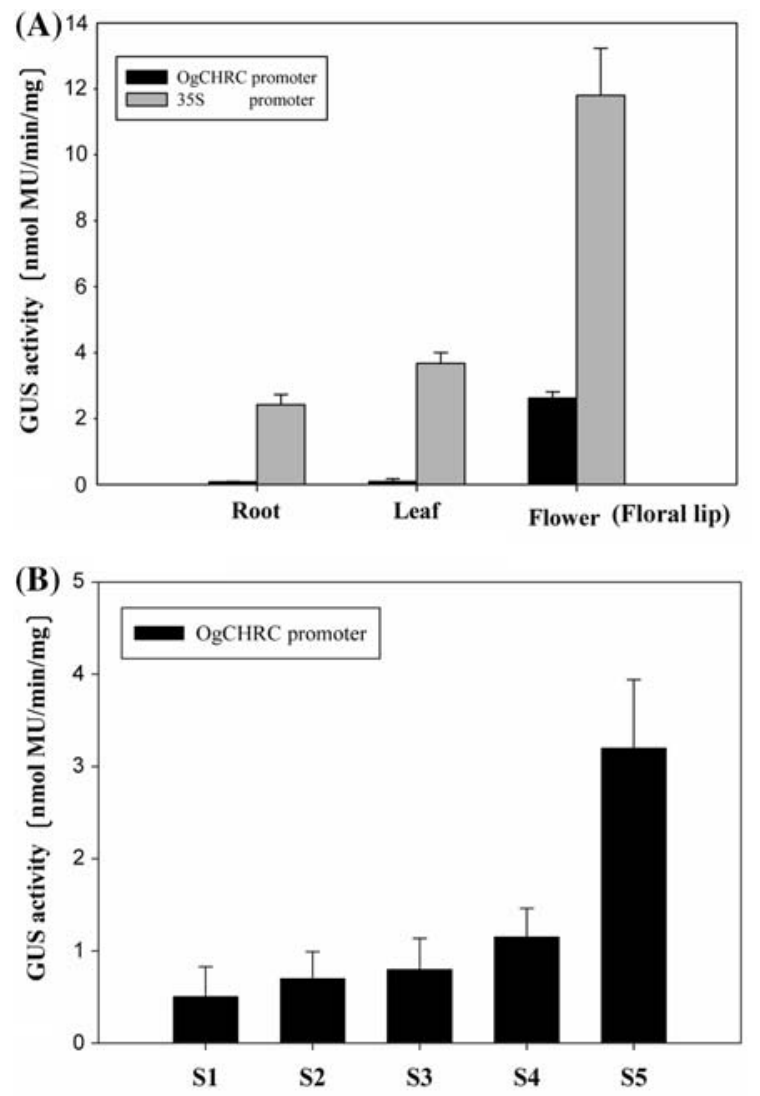

Fig. 3 Spatial and temporal regulation of $O g C H R C$ promoter. (a) GUS activities expressed by Pchrc::GUS and 35S::GUS constructs in transformed tissues were measured and represented as nmol methylumbelliferone (MU) $/ \mathrm{min}^{-1} \mathrm{mg}^{-1}$ protein in the extract. Each result represents an average of six bombardments. (b) GUS activities expressed by Pchrc::GUS delivered by particle bombardment to flower buds of Oncidium at different developmental stages (S1-S5) were measured and represented as nmol methylumbelliferone (MU)/ $\mathrm{min}^{-1} \mathrm{mg}^{-1}$ protein in the extract. Each result represents an average of six bombardments

bombarding a Pchrc::OgMYB construct. It is therefore should be possible to develop new cultivars with different flower colors by regulating chalcone synthase $(C H S)$ and phytoene desaturase $(P T D)$ under the control of a floral-specific promoter, such as the $O g C H R C$ promoter isolated from Oncidium Gower Ramsey (Fig. 5).

Because the CaMV $35 S$ promoter is a constitutive and strong promoter, using it to drive the genes involved in the anthocyanin or carotenoid biosynthetic pathway may result in abnormal growth and development in transgenic plants (Rai et al. 2007;
(A)
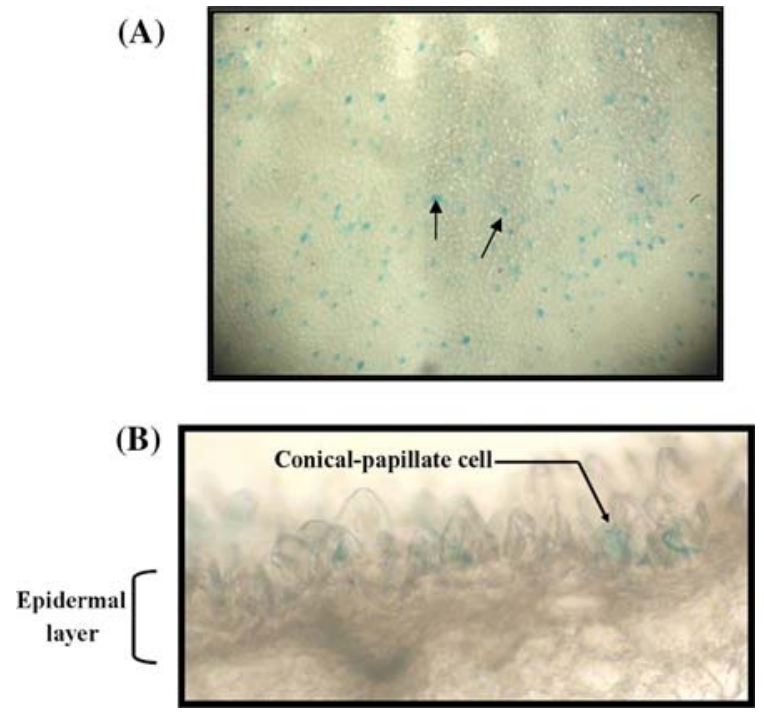

Fig. 4 Tissue-specific and cell-specific expression of Pchrc activity. (a) Histochemical visualization of GUS staining in lip of Oncidium flower bombarded with Pchrc::GUS. (b) Microscopic image of GUS staining in conical-papillate cells of lip tissues bombarded with Pchrc::GUS at magnification of $200 \times$. Scale bars $=50 \mu \mathrm{m}$

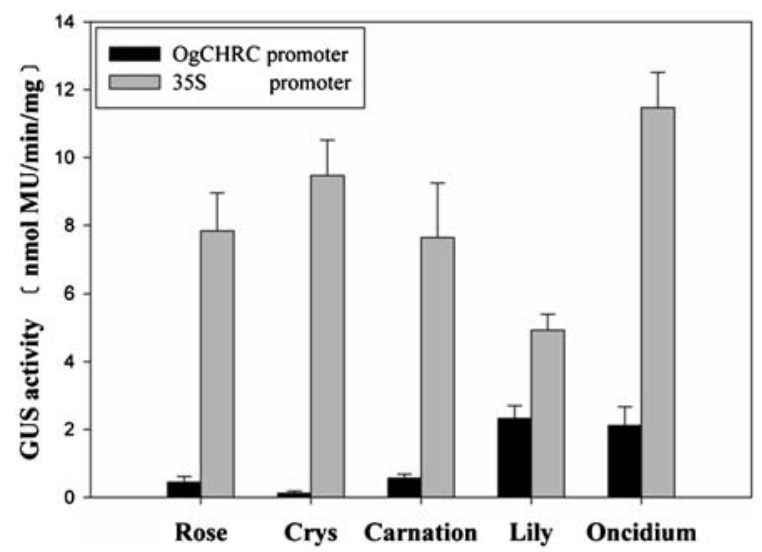

Fig. 5 Pchrc activity assay in petal of different plants. GUS activities expressed by Pchrc::GUS and $35 S:: G U S$ constructs in different plant petals (rose, chrysanthemum, carnation, lily and Oncidium) were measured and represented as nmol methylumbelliferone (MU)/ $\mathrm{min}^{-1} \mathrm{mg}^{-1}$ protein in the extract. Each result represents an average of six bombardments

Van der Meer et al. 1992). The development of new promoters with tissue-specific expression patterns is important for the future development of floriculture crops. The characterization of Pchrc with the spatial and temporal expression may therefore offer a potential application in floral industry. 


\section{References}

Chang S, Puryean J, Cairney J (1993) A simple and efficient method for isolating RNA from pine tree. Plant Mol Biol Rep 11:113-116

Chiou CY, Yeh KW (2008) Differential expression of MYB gene (OgMYB1) determines color patterning in floral tissue of Oncidium Gower Ramsey. Plant Mol Biol 66:379-388

Cooper B, Clarke JD, Budworth P, Kreps J, Hutchison D, Park S, Guimil S, Dunn M, Luginbuhl P, Ellero C, Goff SA, Glazebrook J (2003) A network of rice genes associated with stress response and seed development. Proc Natl Acad Sci USA 100:4945-4950

Frith MC, Hansen U, Weng Z (2001) Detection of cis-element clusters in higher eukaryotic DNA. Bioinformatics 17:878-889

Hieber AD, Mudalige-Jayawickrama RG, Kuehnle AR (2006) Color genes in the orchid Oncidium Gower Ramsey: identification, expression, and potential genetic instability in an interspecific cross. Planta 223:521-531

Higo K, Ugawa Y, Iwamoto M, Korenaga T (1999) Plant cisacting regulatory DNA elements (PLACE) database: 1999. Nucleic Acids Res 27:297-300

Kapoor S, Sugiura M (1999) Identification of two essential sequence elements in the nonconsensus type II PatpB-290 plastid promoter by using plastid transcription extracts from cultured tobacco BY-2 cells. Plant Cell 11:1799-1810

Li SH, Kuoh CS, Chen YH, Chen HH, Chen WH (2005) Osmotic sucrose enhancement of single-cell embryogenesis and transformation efficiency in Oncidium. Plant Cell Tiss Organ Cult 81:183-192

Liau CH, You SJ, Prasad V, Hsiao HH, Lu JC, Yang NS, Chan MT (2003) Agrobacterium tumefaciens-mediated transformation of an Oncidium orchid. Plant Cell Rep 21:993-998

Naik PS, Chanemougasoundharam A, Paul Khurana SM, Kalloo G (2003) Genetic manipulation of carotenoid pathway in higher plants. Curr Sci 85:1423-1430

Prestridge DS (1991) SIGNAL SCAN: a computer program that scans DNA sequences for eukaryotic transcriptional elements. Comput Appl Biosci 7:203-206
Rai M, Datta K, Parkhi V, Tan J, Oliva N, Chawla HS, Datta SK (2007) Variable T-DNA linkage configuration affects inheritance of carotenogenic transgenes and carotenoid accumulation in transgenic indica rice. Plant Cell Rep 26:1221-1231

Stracke R, Ishihara H, Huep G, Barsch A, Mehrtens F, Niehaus K, Weisshaar B (2007) Differential regulation of closely related R2R3-MYB transcription factors controls flavonol accumulation in different parts of the Arabidopsis thaliana seedling. Plant J 50:660-677

Tan J, Wang HL, Yeh KW (2005) Analysis of organ-specific, expressed genes in Oncidium orchid by subtractive expressed sequence tags library. Biotechnol Lett 27:1517-1528

Van der Meer IM, Stam ME, Van Tunen AJ, Mol JN, Stuitje AR (1992) Antisense inhibition of flavonoid biosynthesis in petunia anthers results in male sterility. Plant cell $4: 253-262$

Vishnevetsky M, Ovadis M, Itzhaki H, Levy M, Libal-Weksler Y, Adam Z, Vainstein A (1996) Molecular cloning of a carotenoid-associated protein from Cucumis sativus corollas: homologous genes involved in carotenoid sequestration in chromoplasts. Plant J 10:1111-1118

Vishnevetsky M, Ovadis M, Zuker A, Vainstein A (1999a) Molecular mechanisms underlying carotenogenesis in the chromoplast: multilevel regulation of carotenoid-associated genes. Plant J 20:423-431

Vishnevetsky M, Ovadis M, Vainstein A (1999b) Carotenoid sequestration in plants: the role of carotenoid-associated proteins. Trends Plant Sci 4:232-235

Ytterberg AJ, Peltier JB, van Wijk KJ (2006) Protein profiling of plastoglobules in chloroplasts and chromoplasts. A surprising site for differential accumulation of metabolic enzymes. Plant Physiol 140:984-997

Zuker A, Tzfira T, Ben-Meir H, Ovadis M, Shklarman E, Itzhahi H, Forkmann G, Martens S, Neta-Sharir I, Weiss D, Vainstein A (2002) Modification of flower color and fragrance by antisense suppression of the flavanone 3hydroxylase gene. Mol Breeding 9:33-41 